\title{
Clinical Features and Outcomes of Fusobacterium Species Infections in a Ten-Year Follow-up
}

\author{
Rafael Garcia-Carretero ${ }^{*}$, Marta Lopez-Lomba², Blanca Carrasco-Fernandez², Maria \\ Teresa Duran-Valle ${ }^{2}$ \\ 1 Department of Internal Medicine, Mostoles University Hospital, Madrid, Spain \\ 2 Department of Microbiology, Mostoles University Hospital, Madrid, Spain
}

\begin{abstract}
Objective: Although uncommon, Fusobacterium infections have a wide clinical spectrum, ranging from local pharyngeal infections to septic shock. Our aim was to characterize and analyze the clinical features and outcomes in patients with Fusobacterium infections, and determine which variables were able to predict a poor outcome.

Methods: We conducted a retrospective, hospital-based study using the computerized records of a second-level Spanish general hospital, serving a population of 155,000 inhabitants. The cohort was enrolled among patients cared for at the hospital between 2007 and 2016. Demographic, clinical data, microbiological characterization and outcomes at discharge, were analyzed.

Results: We collected data for all 26 patients over a 10-year period (annual incidence of 1.78 per 100,000), with an incidence of bacteremia of 0.53 cases per 100,000 population per year. F. nucleatum and F. necrophorum were the most frequent isolations ( $53.8 \%$ and $38.5 \%$, respectively). F. necrophorum was found to be associated with a younger population. Although we found no deaths attributable to Fusobacterium, 15 patients (57\%) were found to have severe infections due to this pathogen, and 7 patients (26.9\%) were admitted to the Intensive Care Unit (ICU). The only identifiable risk factor for a severe infection (sepsis, septic shock or ICU admission) was the presence of bacteremia. Conclusions: Fusobacterium infections are uncommon. F. necrophorum tends to cause infection in younger individuals, while F. nucleatum has a preference for older patients. The clinical spectrum is wide, ranging from local, nonsevere infections, such as sinusitis or pharyngitis, to abscess formation and life-threatening infections.
\end{abstract}

Keywords: bacteremia, Fusobacterium, incidence, risk factors, epidemiology, sepsis

Received: 02 October 2017 / Accepted: 13 October 2017

\section{BACKGROUND}

Fusobacterium is an anaerobic gram-negative bacteria which is often involved in the development of Lemierre's syndrome[1], although other bacteria, such as other fusobacteria or Streptococcus, can also be related to this syndrome[2]. The most common species isolated within this genus are F. nucleatum and F. necrophorum. Fusobacterium are usually found as part of the normal oral, gastrointestinal and genital flora, but may induce septic thrombophlebitis in the neighbor- ing neck vessels when the infection is associated with an oropharyngeal abscess. The clinical spectrum may be wide, including abscess formation, bacteremia, puerperal infections, and septic shock[2-6]. Some reliable studies on Fusobacterium $[7,8]$ revealed an overall incidence of bacteremia of 0.55 cases per 100,000 population per year, and a wide spectrum of clinical features. Nohrström et al.[8] categorized the infections into 4 types: Lemierre's syndrome, sepsis in healthy patients, puerperal infections and sepsis in patients with un- 
derlying conditions, in an attempt to identify risk factors, comorbidities and predisposing conditions for favorable outcomes. They reported that some comorbid conditions, such as chronic kidney disease, dementia, cardiovascular disease, diabetes, and ICU admission, may be considered as risk factors for poor outcomes. Our aim was to characterize and analyze the clinical features and outcomes of patients with Fusobacterium infections, as well as predisposing diseases, and determine which variables were able to predict favorable or poor outcomes.

\section{- METHODS}

\section{Study design}

We conducted a retrospective cohort study at the Mostoles University Hospital in Madrid, a second-level general hospital serving a high variety of patients, and a teaching hospital for specializing postgraduates. The cohort was enrolled among patients cared for at the hospital between 2007 and 2016 (9.4 years), and they were evaluated retrospectively. All patients with an isolation of Fusobacterium species were identified in that timeframe through our Clinical Microbiology Laboratory, which is the only laboratory processing the clinical specimens in our area. We considered the patient to be suitable for entering the study if, along with the isolation of the fusobacteria, he/she exhibited clinical characteristics of an ongoing systemic infection. Thanks to our electronic record system, we were able to collect data regarding demographic features such as age and gender, clinical characteristics such as comorbidities, malignancy or immunocompromised status, hospital stay, medical or surgical management, and outcome. Sepsis was defined according to The Third International Consensus Definitions for Sepsis and Septic Shock[9]. Therefore, organ dysfunction was identified as an acute change in total SOFA (Sequential [Sepsis-Related] Organ Failure Assessment) score $\geq 2$ points consequent to the infection caused by Fusobacterium species. Sepsis diagnosis was established if the patient met the organ dysfunction criteria and qSOFA (quick SOFA) score $\geq 2$ of the following items: alteration in mental status, systolic blood pressure $\geq 100 \mathrm{~mm} \mathrm{Hg}$, or respiratory rate $\geq 22 / \min [10,9]$.

Septic shock was defined by the presence of sepsis with persisting hypotension requiring vasopressors to maintain mean arterial pressure $\geq 65 \mathrm{~mm} \mathrm{Hg}$ and hav- ing a serum lactate level $>2 \mathrm{mmol} / \mathrm{L}(18 \mathrm{mg} / \mathrm{dL})$ despite adequate fluid resuscitation. We defined severe infection as sepsis, need for ICU admission, bacteremia, peritonitis, disseminated intravascular coagulation, pelvic inflammatory disease, and septic shock. We were able to identify the source of infection thanks to the electronic records, imaging studies and microbiological isolations within the tissues.

\section{Microbiological identification}

Species-level identification was performed from positive cultures by manual methods, by means of the commercial system of multi-substrate analytical profile index, API 20A, that determines the phenotypical characteristics for anaerobic bacteria identification, both gram-negative and gram-positive. First isolations in our cohort were identified by automated methods-i. e. mass spectrometry Matrix-Assisted Laser Desorption Ionization Time Of Flight (MALDI-TOF MS)[11,12].

\section{Statistical analysis}

We performed a normality test for continuous variables using the Shapiro-Wilk test to assess the shape of the distribution of the continuous variables. Data are reported as the median, interquartile range (IQR) and percentage. We used either the Chi-square test or Fisher's exact test to assess the comparison between categorical variables, the Mann-Whitney-Wilcoxon test to establish comparisons between continuous and categorical variables, and Spearman's correlations to compare 2 continuous variables. Statistical analysis was performed using R version 3.3.2 (2016-10-31)[13]. For statistical comparison, a p-value of 0.05 was considered significant.

\section{DESULTS}

\section{Demographic characteristics}

We collected clinical records and microbiological data for 26 patients, of whom 9 were women (34.6\%) and 17 were men $(65.4 \%)$. The demographic features are summarized in Table 1. The median age was 46.5 years (IQR, 20.5 years). Four patients (15.4\%) were active smokers. In terms of comorbidities, 4 patients (15.4\%) had respiratory diseases (asthma or chronic obstructive pulmonary disease), and 4 patients (15.4\%) had colorectal cancer, either prior to the Fusobacterium infection or diagnosed during the workup. Only 2 pa- 
tients were considered to have immunocompromised status, due to either active HIV infection or chemotherapy treatment.

\section{Presentation, sources of infection and outcome}

Clinical characteristics and outcomes are shown in Table 1 . Sepsis was present in 9 patients (34.6\%). Only 4 patients (15.4\%) developed severe complications: peritonitis (2 patients), disseminated intravascular coagulation (1 patient), and pelvic inflammatory disease (1 patient). However, septic shock could not be identified in any patient. An intra-abdominal source of the infection was identified in 12 patients (46.1\%), while an upper respiratory tract source was identified in 5 patients (19.2\%), and a lower respiratory tract source in 2 patients (7.7\%). None of the patients presented with Lemierre's syndrome. All 3 sigmoid colon abscesses were related to colorectal cancer, while 19 (73.1\%) out of 26 patients were diagnosed with an abscess of any origin. Surgical management, whether open surgery, drainage or aspiration, was required in 18 patients (69.2\%). While most of the patients stayed at the hospitalization ward, only 7 patients (26.9\%) had to be admitted to the Intensive Care Unit (ICU). The median length of hospital stay was 11.5 days (IQR, 21 days). All 26 patients survived the Fusobacterium infection. Those who died months or years later could not be attributable to bacterial infections.

\section{Microbiological features}

Sample specimens were obtained from exudate (57.7\%), blood (30.8\%) and tissue (11.5\%). Data regarding microbiological characteristics are summarized in Table 2. Fusobacterium necrophorum and F. nucleatum were the most frequent isolations (92.3\%). One case of $F$. mortiferum was identified, and only one Fusobacterium strain could not be identified. The annual incidence of fusobacterial infection was 1.78 per 100,000 population. Although the median age was very similar in both F. nucleatum (median 49.5 years, IQR 18.25) and F. necrophorum (median 40.5 years, IQR 35.25 ), the incidence follows a pattern in which F. necrophorum affects a younger population (Figure 1).

The isolation of bacteria in pure culture was more frequent $(80.8 \%)$ than in the polymicrobial culture (19.2\%). Strains of Bacteroides fragilis, Escherichia coli, Klebsiella pneumoniae and Enterococcus faecium were identified in the polymicrobial cultures.

\section{Antimicrobial susceptibility testing}

Data regarding antimicrobial susceptibility were available in all 26 patients. All strains were susceptible to metronidazole, clindamycin, piperacillin/tazobactam and carbapenems. Resistance to penicillin was present in 16 isolates ( 9 cases of $F$. nucleatum and 7 cases of $F$. necrophorum).

Table 1. Demographic and clinical features, and outcomes of the studied population

\begin{tabular}{lc} 
Characteristics & Patients \\
\hline Total Patients & 26 \\
Age (years) & $46.5(20.5)$ \\
Gender (female) & $9(34.6 \%)$ \\
Gender (male) & $17(65.4 \%)$ \\
Asthma/COPD & $4(15.4 \%)$ \\
Smoking habit & $4(15.4 \%)$ \\
Colorectal cancer & $4(15.4 \%)$ \\
Immunocompromised status & $2(7.7 \%)$ \\
Clinical features & \\
Sepsis & $9(34.6 \%)$ \\
Sources of infection & \\
Peritonsillar abscess & $6(23.1 \%)$ \\
Perforated acute appendicitis & $3(11.5 \%)$ \\
Liver abscess & $2(7.7 \%)$ \\
Ovarian abscess & $2(7.7 \%)$ \\
Chorioamnionitis & $2(7.7 \%)$ \\
Empyema & $3(11.5 \%)$ \\
Sigmoid colon abscess & $3(11.5 \%)$ \\
Sinusitis & $2(7.7 \%)$ \\
Other complications & $4(15.4 \%)$ \\
ICU admission & $7(26.9 \%)$ \\
Surgical treatment & $18(69.2 \%)$ \\
\hline
\end{tabular}

Date are given in number and percentages, except for age, which is given in median and interquartile range. COPD: chronic obstrutive pulmonary disease. Other complications: peritonitis, disseminated intravascular coagulation and pelvic inflammatory disease.

Table 2. Microbiological characteristics of the studied population

\begin{tabular}{lc} 
Sample features & $\begin{array}{c}\text { Sample size } \\
\text { (percentage) }\end{array}$ \\
\hline Exudate sample (\%) & $15(57.7 \%)$ \\
Blood sample (\%) & $8(30.8 \%)$ \\
Tissue sample (\%) & $3(11.5 \%)$ \\
Species isolated & \\
Fusobacterium mortiferum & $1(3.8 \%)$ \\
Fusobacterium necrophorum & $10(38.5 \%)$ \\
Fusobacterium nucleatum & $14(53.8 \%)$ \\
Fusobacterium sp & $1(3.8 \%)$ \\
Isolation in pure culture & $21(80.8 \%)$ \\
\hline
\end{tabular}


144 - The Journal of Critical Care Medicine 2017;3(4)

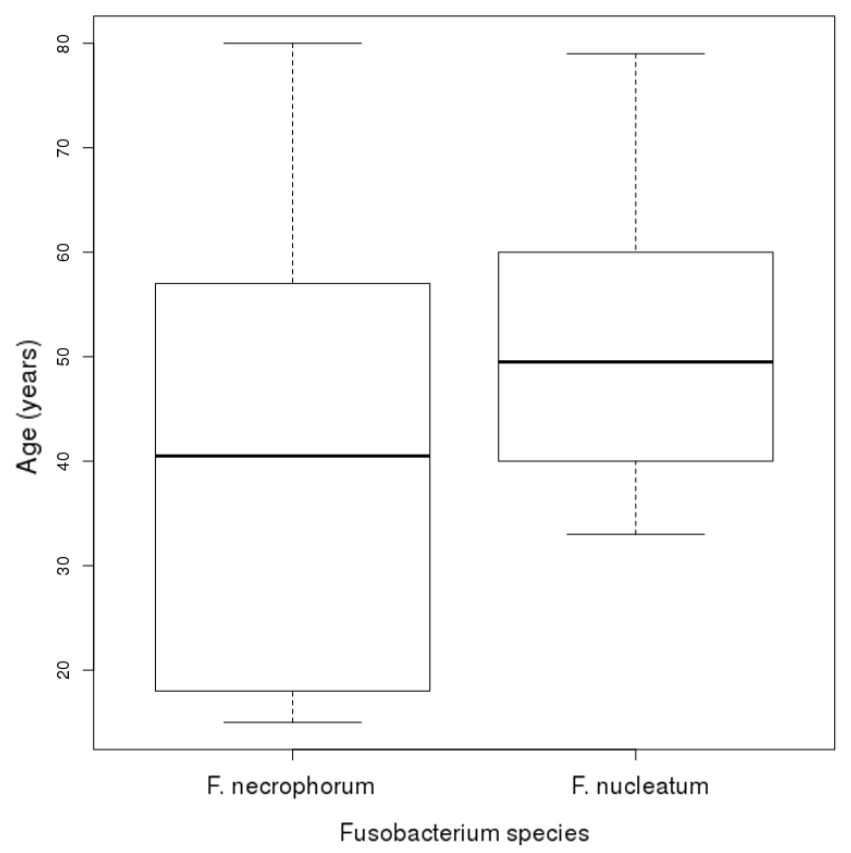

Fig. 1. Boxplot showing age distribution by Fusobacterium species

\section{Risk factors for severe disease}

The univariate analysis of the risk factors for severe infection is shown in Table 3. We found no significant differences in terms of gender, age, isolated species, presence of an abscess, immunosuppression status, presence of a tumor or respiratory disease between the patients presenting with severe infection and those with non-severe features. However, bacteremia (Chisquared $=6.1553, \mathrm{df}=1, \mathrm{p}$-value $=0.0073$ ) was statistically significant. When included in a linear regression analysis, bacteremia could be considered as an independent risk factor for severe infection (OR 0.611, 95\% CI $0.240-0.981, \mathrm{p}=0.002$ ).

\section{Risk factors for length of hospital stay}

After performing a univariate analysis of the risk factor for longer hospital stay, we could not find significant
Available online at: www.jccm.ro

differences for length of hospital stay with regard to gender, age, Fusobacterium species, bacteremia, presence of an abscess, immunosuppression condition, malignant tumor, or respiratory disease.

\section{DISCUSSION}

The main findings in our case series showed the wide spectrum of clinical presentations caused by Fusobacterium infections, ranging from local pharyngeal or upper respiratory tract infections, such as sinusitis, to life-threatening infections that require ICU admission. Despite the severe infection, after appropriate management with antibiotic therapy or surgical procedures if required, patients were successfully discharged. Fusobacterium is an anaerobic pathogen usually involved in the development of Lemierre's syndrome[1], although other bacteria may also cause this complication of pharyngitis. Typical manifestations of Lemierre's syndrome include the development of a pyogenic thrombosis of the internal jugular vein, and septic emboli to the lungs, brain, and liver[2]. However, in our series we found no cases of Lemierre's syndrome, unlike some other case series which did identify the syndrome. Nevertheless, Lemierre's syndrome is an uncommon infection[2], with an annual incidence of between 0.05 and 0.09 per 100,000 population[2,8], and like ours, there are other case series which did not find any cases presenting this syndrome[14].

Fusobacterium infections, whether soft-tissue or bacteremic infections, are uncommon. During the 10 -year period of observation, we found 26 cases of fusobacterial infections in a population of 155,000 inhabitants. The annual incidence, according to our retrospective study, was 1.78 per 100,000 population, and the incidence of bacteremia was 0.53 per 100,000 population, which is very similar to other retrospective studies: Nohrström et al.[8] reported an annual incidence of

Table 3. Univariate analysis of the risk factors for severe infection

\begin{tabular}{|c|c|c|c|}
\hline Variable & Severe infection $(n=15)$ & Non-severe infection $(n=11)$ & p-value \\
\hline Gender (male) & $10(66.7 \%)$ & $7(46.69 \%)$ & 1 \\
\hline Age (years) & $49+/-22$ & $42+/-15.9$ & 0.420 \\
\hline F. nucleatum & $8(53.36 \%)$ & $6(40.02 \%)$ & 1 \\
\hline F. necrophorum & $7(46.69 \%)$ & $3(20.01 \%)$ & 0.551 \\
\hline Bacteremia & $8(53.36 \%)$ & 0 & 0.007 \\
\hline Abscess & $10(66.7 \%)$ & $9(60.93 \%)$ & 0.679 \\
\hline Immunosuppression & $3(20.01 \%)$ & 0 & 0.238 \\
\hline Cancer & $3(20.01 \%)$ & $1(6.67 \%)$ & 0.613 \\
\hline Respiratory disease & $1(6.67 \%)$ & $3(20.01 \%)$ & 0.374 \\
\hline
\end{tabular}


bacteremia of 0.55 per 100,000 population, and the series by Afra et al.[7] also reported an incidence of 0.55 per 100,000 per year. Pett et al.[15] reported an incidence of 0.76 cases per 100,000 per year.

An interesting feature was the preference F. necrophorum showed for healthy, younger patients. F. nucleatum cases had a median age of 49.5 years (IQR 18.25), while F. necrophorum had a median of 40.5 years (IQR 35.25). Although the median only differs by 9 years, Figure 1 shows that $F$. nucleatum did not affect individuals under 40 years of age, while F. necrophorum affected individuals up to 18 years of age. Other studies are more consistent with these findings, such as that by Afra et al.[7], who reported an obvious pattern between F. necrophorum (median of 21 years) and F. nucleatum (median of 53.5 years), and Pett et al.[15], who reported that $F$. necrophorum affected patients under 47 years of age.

We also observed a male predominance (65.4\%) versus female infections (34.6\%). These findings were consistent with other publications $[7,15]$.

$F$. necrophorum and $F$. nucleatum were the most common isolated species in our study (24 out of 26 patients), consistent with other retrospective studies $[2,7,15]$. We only reported one strain of $F$. mortifer$u m$, and one strain of unidentified Fusobacterium. Other studies, however, reported the isolation of $F$. varium and F. gonidiaformans[15].

In addition to the above-mentioned low likelihood of causing bacteremic infections, Fusobacterium species tend to involve abscess formation (17 patients out of our 26 reported cases), perhaps due to an attempt to protect an anaerobic environment from the oxidative conditions of the host. In our series, not all abscesses required surgical treatment: one patient presenting with a liver abscess was managed with antibiotic therapy only, with a good outcome at discharge. In our study, all abscesses were considered to arise from primary infections, except the 2 cases of liver abscesses, the primary source of which was probably the disruption of the mucosal colonic barriers related to colorectal cancer. However, some publications have reported liver abscessation related to periodontal disease[6] without Lemierre's syndrome, or after a dental procedure[16] (dental cleaning in an otherwise healthy patient). Colorectal tumors, diverticular disease and even a recent colonoscopy[17] may be the potential source of Fusobacterium infection. A recent publication reported a liver abscess in an immunocompetent patient with no recognizable risk factors for Fusobacterium infection[18].

The liver is the most common visceral organ in which abscesses can be found, but very few cases of Fusobacterium as a causative pathogen have been reported. With regard to Lemierre's syndrome, about $2-4 \%$ of cases develop liver abscesses when metastatic emboli occur[2]. Liver abscesses are often polymicrobial, due to a complication of peritonitis, spreading from a biliary infection or via hematogenous spread in the context of a systemic infection.[5] Other risk factors for a liver abscess include underlying gastrointestinal neoplasm, such as colorectal or hepatobiliary carcinoma, pancreatic abscesses, diabetes and a certain degree of immunosuppression[3]. Although the liver is the most common site of metastatic visceral abscesses, the presence of septic emboli involving this organ is uncommon. With no evidence of pharyngeal infection, it is less common to diagnose a liver abscess in an immunocompetent patient, and a workup should therefore be performed in order to identify the primary focus of the infection $[5,7]$.

In our series, malignancy, in the form of colorectal cancer, was found in 4 patients (15.4\%), associated with F. nucleatum in 2 patients, and F. necrophorum in the other 2 cases. Three patients with this underlying condition showed features of severe infection, and the need for ICU admission. Only one patient presented with a solitary liver abscess, and the malignancy was diagnosed in the ensuing workup. Several studies have reported the association between malignancy and Fusobacterium infections[7,8,14,19], although other comorbidities such as diabetes, dementia, respiratory disease, and heart disease may also be risk factors[7]. In our study, however, despite the fact that malignancy, chronic obstructive pulmonary disease, and immunosuppressed status were encoded in our electronic dataset, we could not find any statistical association between these risk factors and the likelihood of severe infection. The only identifiable risk factor for a severe infection (sepsis or ICU admission) was the presence of bacteremia.

Regarding the outcomes, we were able to report a low mortality due to Fusobacterium infections since we did not record any deaths attributable to this pathogen. These data are not consistent with those obtained by other publications, which report a mortality rate of $1 \%$,[20] $11 \%$,[7] or even $47.4 \%[21]$.

Our study has a number of limitations. Although patient data were available, given the uncommon na- 
ture of these infections, even in a 10 -year observation period, the size of our cohort remained small (26 patients), in contrast with other aforementioned publications. For this reason, we believe our results regarding risk factors lack statistical significance. With further studies, researchers should be able to shed light on the mechanisms of infection, underlying conditions, and the risk factors involving Fusobacterium infections.

\section{- CONCLUSIONS}

Fusobacterium species are the causative pathogen of uncommon infections. The overall incidence found in our study is consistent with other publications. F. necrophorum tends to cause infection in younger individuals, while F. nucleatum has a preference for older patients. The clinical spectrum is wide, ranging from upper respiratory tract infections, such as sinusitis or pharyngitis, to abscess formation and septic shock. Until sample cultures can yield any definitive results, empirical treatment with broad-spectrum antibiotics, such as metronidazole or carbapenems, should be initiated. This treatment should be corrected according to sensitivities and drug resistances. The elective treatment for an abscess is usually percutaneous or surgical drainage, although in some cases, broad-spectrum, systemic antibiotics and medical management may be enough to achieve the complete resolution of the abscess.

\section{CONFLICT OF INTEREST}

The authors declare that they have no conflict of interest.

\section{ACKNOWLEDGMENTS}

Authors would like to thank Jacqueline Lamb for her invaluable advice and tips for English grammar and spelling, and Blanca San Jose Montano, the Health Science Librarian-Documentalist of our institution, for her great support, suggestions and encouragement in the making of this manuscript.

\section{ETHICAL APPROVAL}

All procedures performed in studies involving human participants were in accordance with the ethical standards of the institutional and/or national research committee and with the 1964 Helsinki declaration and its later amendments or comparable ethical standards. Informed consent: For this type of study formal consent is not required.

\section{REFERENCES}

1. Lemierre A. On certain septicaemias due to anaerobic organisms. Lancet. 1936;227(5874):701-3.

2. Riordan T. Human infection with Fusobacterium necrophorum (Necrobacillosis), with a focus on Lemierre's syndrome. Clin Microbiol Rev. 2007 Oct;20(4):622-59.

3. Rahimian J, Wilson T, Oram V, Holzman RS. Pyogenic liver abscess: recent trends in etiology and mortality. Clin Infect Dis. 2004 Dec;39(11):1654-9.

4. Iwasaki T, Yamamoto T, Inoue K, Takaku K. A Case of Lemierre's Syndrome in Association with Liver Abscess without Any Other Metastatic Lesions. Intern Med. 2012;51(11):1419-23.

5. Kaplan GG, Gregson DB, Laupland KB. Population-based study of the epidemiology of and the risk factors for pyogenic liver abscess. Clin Gastroenterol Hepatol. 2004 Nov;2(11):1032-8.

6. Yoneda M, Kato S, Mawatari H, Kirikoshi H, Imajo K, Fujita K, et al. Liver abscess caused by periodontal bacterial infection with Fusobacterium necrophorum. Hepatol Res. 2011 Feb;41(2):194-6.

7. Afra K, Laupland K, Leal J, Lloyd T, Gregson D. Incidence, risk factors, and outcomes of Fusobacterium species bacteremia. BMC Infect Dis. 2013;13(1):264.

8. Nohrström E, Mattila T, Pettilä V, Kuusela P, Carlson P, Kentala $E$, et al. Clinical spectrum of bacteraemic Fusobacterium infections: from septic shock to nosocomial bacteraemia. Scand J Infect Dis. 2011;43(6-7):463-70.

9. Singer $M$, Deutschman CS, Seymour CW, Shankar-Hari $M$, Annane D, Bauer $M$, et al. The Third International Consensus Definitions for Sepsis and Septic Shock (Sepsis-3). JAMA. 2016 Feb;315(8):801-10.

10. Shankar-Hari M, Phillips GS, Levy ML, Seymour CW, Liu VX, Deutschman CS, et al. Developing a new definition and assessing new clinical criteria for septic shock: for the Third International Consensus Definitions for Sepsis and Septic Shock (Sepsis-3). Jama. 2016;315(8):775-87.

11. O'Hara CM. Manual and Automated Instrumentation for Identification of Enterobacteriaceae and Other Aerobic GramNegative Bacilli. Clin Microbiol Rev. 2005 Jan;18(1):147-62.

12. Kierzkowska M, Majewska A, Kuthan RT, Sawicka-Grzelak A, Mlynarczyk G. A comparison of Api 20A vs MALDI-TOF MS for routine identification of clinically significant anaerobic bacterial strains to the species level. J Microbiol Methods. 2013 Feb;92(2):209-12.

13. R Core Team. R: A Language and Environment for Statistical Computing. Vienna, Austria; 2014.

14. GoldbergEA, Venkat-RamaniT, HewitM, Bonilla HF. Epidemiology and clinical outcomes of patients with Fusobacterium 
Available online at: www.jccm.ro

bacteraemia. Epidemiol Infect. 2013;141(2):325-9.

15. Pett E, Saeed K, Dryden M. Fusobacterium species infections: clinical spectrum and outcomes at a district general hospital. Infection. 2014;42(2):363-70.

16. Bytyci F, Khromenko E. Hepatic abscess caused by Fusobacterium necrophorum after a trip to the dentist. BMJ Case Rep. 2016 Mar;2016.

17. Buelow BD, Lambert JM, Gill RM. Fusobacterium liver abscess. Case Rep Gastroenterol. 2013;7(3):482-6.

18. Ahmed Z, Bansal SK, Dhillon S. Pyogenic liver abscess caused by Fusobacterium in a 21-year-old immunocompetent male.
The Journal of Critical Care Medicine 2017;3(4) • 147

World J Gastroenterol. 2015 Mar 28;21(12):3731-5.

19. Li Y-Y, Ge Q-X, Cao J, Zhou Y-J, Du Y-L, Shen B, et al. Association of Fusobacterium nucleatum infection with colorectal cancer in Chinese patients. World J Gastroenterol. 2016;22(11):3227.

20. Huggan PJ, Murdoch DR. Fusobacterial infections: clinical spectrum and incidence of invasive disease. J Infect. 2008;57(4):283-9.

21. Yang C-C, Ye J-J, Hsu P-C, Chang H-J, Cheng C-W, Leu H-S, et al. Characteristics and outcomes of Fusobacterium nucleatum bacteremia-a 6-year experience at a tertiary care hospital in northern Taiwan. Diagn Microbiol Infect Dis. 2011;70(2):167-74. 